\title{
Spatial Integration Grids in Line Profile Modelling of Nonradially Pulsating Magnetic Stars
}

\author{
R.M. Fensl, G. Könighofer, M.J. Stift
}

Institut für Astronomie, Türkenschanzstr. 17, Wien, Austria

\section{The philosophy of the resolution function}

For an optimum grid we postulate a fractional change of the emergent Stokes parameters between two adjacent surface points not to exceed some fixed value (say $20 \%$ ) at any frequency point. The contribution from a local Stokes IQUV profile to the observed integrated profile is a complicated non-linear function of rotational and pulsational Doppler shifts respectively, of Zeeman splitting, magnetic field direction relative to the line-of-sight, of the magnetic field azimuth in the observer's frame and of limb darkening. The run of IQUV with visible disk coordinates $(x, y)$ can be represented by curved surfaces $f(x, y)$; these are different for each Stokes parameter and frequency point. The optimum integration grid then has to ensure that the maximum change $\Delta f(x, y)$ over all frequencies and Stokes parameters does not exceed the above-mentioned limit.

Expressing the average fractional change in the value of the Voigt function by an average change in normalised (to the Doppler width of the line) wavelength difference, we quadratically sum up the individual contributions to the total difference in adjacent $I Q U V$ intensities as enumerated above to obtain a piecewise resolution function. Inverse equidistant interpolation in the cumulative resolution function yields the desired optimum grid (for details see Fensl 1995).

\section{Application to the Oblique Pulsator model and conclusions}

The Oblique Pulsator (Kurtz 1990) explains the observed short-term variations (4-15 $\mathrm{min}$ ) of roAp stars by invoking non-radial pulsations of low degree $l$ and of order $m=0$; the pulsation axis is aligned with the magnetic axis which is itself inclined at a non-zero angle to the rotation axis. We have made calculations for this simple geometry with a code based on Stift's (1985) Stokes code but including linear polarisation and magneto-optical effects. The code is written in Ada83 making the manipulation of Stokes, magnetic and coordinate vectors, and of transformation matrices particularly easy and transparent (operator overloading, reusable packages).

Fig. 1 (top row) shows Stokes IQUV profile for three different models at three different rotational phases (low degree zonal modes $l \leq 5, m=0$ only). Note the wealth of non-spurious structure in $Q U V$, perfectly resolved with 260 grid points! On account of the simple geometry of the problem the improvement over fixed grids is not overly dramatic but we find that the excellent accuracy obtained with our grid - measured by $\Sigma$ the square root of the cumulated variances of the deviations in IQUV from the reference profile - is always maintained 


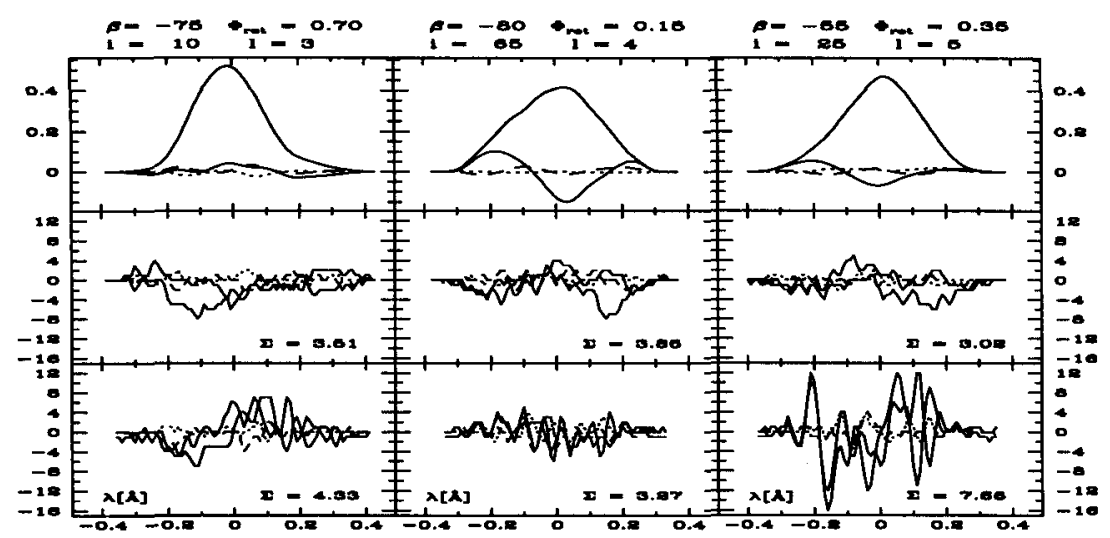

Figure 1. Top: IQUV profiles (full, dashed, dotted, full) for a Fe II $\lambda 6150$ line, assuming a Zeeman triplet, $v \sin i=7 \mathrm{~km} / \mathrm{s}, v_{\text {puls }}=$ $10 \mathrm{~km} / \mathrm{s}, \Phi_{\text {puls }}=0.0, H_{\text {pole }}=0.4 \mathrm{~T}$ and using a $(9000,4.0,0.0) \mathrm{Kurucz}$ atmosphere. Inclination $i$, obliquity $\beta$, pulsation degree $l$ (with $m=0$ ) and rotational phase $\Phi_{\text {rot }}$ are given above. Middle: Differences between profiles calculated with 260 adaptive points and reference profiles (7000 points); 100 times exaggerated scale. IQ (full, dotted) above, $U V$ (dotted, full) below. Bottom: Same for fixed grid of 204 equally sized visible elements. For $\Sigma$ the numerical accuracy in $10^{-4}$ see text.

(middle rows). Fixed grids can sometimes yield comparable results but one can never rely on that (bottom rows).

The main advantage of adaptive grids lies in the guaranteed resolution without the need to provide for huge safety margins in the number of grid points. Additionally, in the large majority of cases - particularly those with more complex magnetic geometries - we obtain considerable gains in accuracy at constant grid size and CPU time. This becomes possible only because the grid adapts to the velocity and magnetic field distributions particular to the magnetic geometry and pulsational pattern at a given rotational and pulsational phase. Our extension of the method of Stift (1985) proves to deal very effectively and accurately with linear polarisation and with non-radial pulsation. We would contend that at present adaptive grids as outlined above constitute the best way to guarantee reliable and accurate Stokes profile modelling in the presence of macroscopic velocity and of inhomogeneous magnetic fields, using a minimum of computational resources.

An Ada83 package for establishing an adaptive grid is available upon request from stift@astro.ast.univie.ac.at.

\section{References}

Fensl, R.M. 1995, A\&A, in press

Kurtz, D.W. 1990, ARA\&A, 28, 607

Stift, M.J. 1985, MNRAS, 217, 55 